\title{
Evaluation of the Image Quality of Ink-Jet Printed Paper Copies of Digital Chest Radiographs as Compared With Film: A Receiver Operating Characteristic Study
}

\author{
Kerstin Lyttkens, Tomas Kirkhorn, Mikael Kehler, Berth Andersson, Anders Ebbesen, Peter Hochbergs, \\ Olof Jarlman, Claes-Göran Lindberg, and Nils-Gunnar Holmer
}

Paper copies of digital radiographs printed with the continuous ink-jet technique have proved to be of a high enough quality for demonstration purposes. We present a study on the image quality of ink-jet printed paper copies of digital chest radiographs, based on receiver operating characteristic (ROC) analysis. Eightythree digital radiographs of a chest phantom with simulated tumors in the mediastinum and right lung. derived from a computed radiography (CR) system were presented in two series of hard copies as ink-jet printed paper copies and as laser recorded film. The images, with a matrix of $1,760 \times 2,140$ pixels, were printed with a spatial resolution of 10 pixels $/ \mathrm{mm}$ in the CR film recorder as well as in the ink-jet printer. On film, every image was recorded in two versions, one optimized for the mediastinum and one for the lungs. On paper, only one image was printed; this constituted an effort to optimize both the mediastinum and the lungs. The ink-jet printed images, printed on a matt coated paper, were viewed as on-sight images with reflected light. The examinations were reviewed by six radiologists, and $R O C$ curves were constructed. No significant difference was found between the performance of film and that of ink-jet paper prints. Because the cost for a paper copy is only a tenth of that of film, remarkable cost reductions can be achieved by using the ink jet technique instead. Our results show that further quality studies of ink-jet printed images are worthwhile.

Copyright 1994 by W.B. Saunders Company

KEY WORDS: medical imaging; hard copy; ink-jet printing; radiography digital, receiver operating characteristics (ROC), thorax, phantom.

$A$ $S$ A CONSEQUENCE of the introduction of digital systems in radiology, the traditional diagnostic work with film will probably be abandoned in favor of work at monitor-based viewing stations. Even if interpretation on monitors makes film superfluous as a medium for diagnostics, it is implausible to assume that every radiologist, or other user, may have access to a digital viewing system. Some kind of hard copy for demonstration purposes will therefore be needed. As an alternative to the expensive film, we are investigating the possibility of using ink-jet printed paper copies for this purpose.

In a previous pilot study, we have found the image quality of paper prints printed with a continuous ink-jet technique high enough for demonstration. ${ }^{1}$ The objective of this study was to determine if ink-jet paper copies are sufficient for detection of nodules in the lung and the mediastinum. Digital chest radiographs of a phantom with randomly placed nodules in the mediastinum and the lung were studied. The radiographs were printed by the ink-jet printer, and the image quality was compared with that of corresponding film prints, with a receiver operating characteristic (ROC) analysis. ${ }^{2}$

\section{MATERIALS AND METHODS}

\section{Phantom}

An anthropomorphic chest phantom (Humanoid systems, Carson, CA) was used, with test objects simulating tumors applied to the phantom. The phantom consisted of heart, lungs and thoracic cage in a torso made of a tissueequivalent material. At the back of the phantom was a grid containing 27 squares in the mediastinum and 110 squares in the right lung. The test objects simulating tumors were four hemispheres consisting of acetal plastic (Delrin), two with a diameter of $5 \mathrm{~mm}$, and one each of diameters 10 and $15 \mathrm{~mm}$, respectively. The presence-including localization in a square-or the absence for each object, independent of the others, was randomized by a computer. In addition to these test objects, other objects were present in the mediastinum and both lungs. Because they had a fixed position, they were of no use for the study, nor were they a source of error.

From the Department of Diagnostic Radiology, Lund University Hospital; the Department of Electrical Measurements, Lund Institute of Technology, and the Department of Biomedical Engineering, Lund University, Lund, Sweden.

Address reprint requests to Kerstin Lyttkens, MD, Department of Diagnostic Radiology, Lund University Hospital, S-221 85 Lund, Sweden.

Copyright 11994 by W.B. Saunders Company

0897-1889/94/0702-0012\$03.00/0 


\section{Image Acquisition}

The digital radiographs were produced with a computed radiography (CR) system, Digiscan (Siemens AG, Medical Engineering Group, Erlangen, Germany), using photostimulable phosphor plates, ${ }^{3,4}$ Fuji ST-III, $35 \times 43 \mathrm{~cm}^{2}$ (Fuji Photo Film Company, Tokyo, Japan).

Eighty-three images were obtained in the posteroanterior projection using a movable grid (36 lines $/ \mathrm{cm}$; ratio, $12: 1$ ). The images were exposed at $90 \mathrm{kV}$ and $20 \mathrm{~mA}$ (film-focus distance, $100 \mathrm{~cm}$; focus size, $0.6 \mathrm{~mm}$ ). Two small lead markers, placed on the back and front of the phantom, helped to position it similarly in all exposures.

The images, with a matrix of $1,760 \times 2,140$ pixels, each pixel represented by 10 bits, were stored on an optical disc as raw data, ie, without any image processing applied.

\section{Film Hard Copies}

Two different images were recorded on film from one exposure. One was optimized in density range for the mediastinum and one for the lungs. In addition, both images were edge- enhanced by an unsharp mask algorithm. On each film, with a size of $257 \times 364 \mathrm{~mm}^{2}$, two images of $176 \times 214 \mathrm{~mm}^{2}$ each was printed with a spatial resolution of 10 pixels $/ \mathrm{mm}^{3}$

\section{Ink-Jet Printer Technique}

The ink-jet printer, which is based on the Hertz' continuous ink-jet technique, was developed at the Department of Electrical Measurements, Lund Institute of Technology, Lund, Sweden. ${ }^{5}$ The technique has been described earlier in detail. ${ }^{1,6}$ Figure 1 shows the principle of the technique in which a continuous ink jet is generated by forcing ink under high pressure through a $10-\mu \mathrm{m}$ glass nozzle. When the nozzle is stimulated with a piezo-electric crystal, the jet disintegrates into separate and equally sized drops at a rate of 1 million drops per sec. The continuous train of ink drops can be controlled by electric charging and deflection. With a control electrode at the point of drop formation, each formed drop can be charged or left uncharged. Charged drops will be deflected in a deflection field and caught below a knife edge. The uncharged drops will fly undisturbed straight

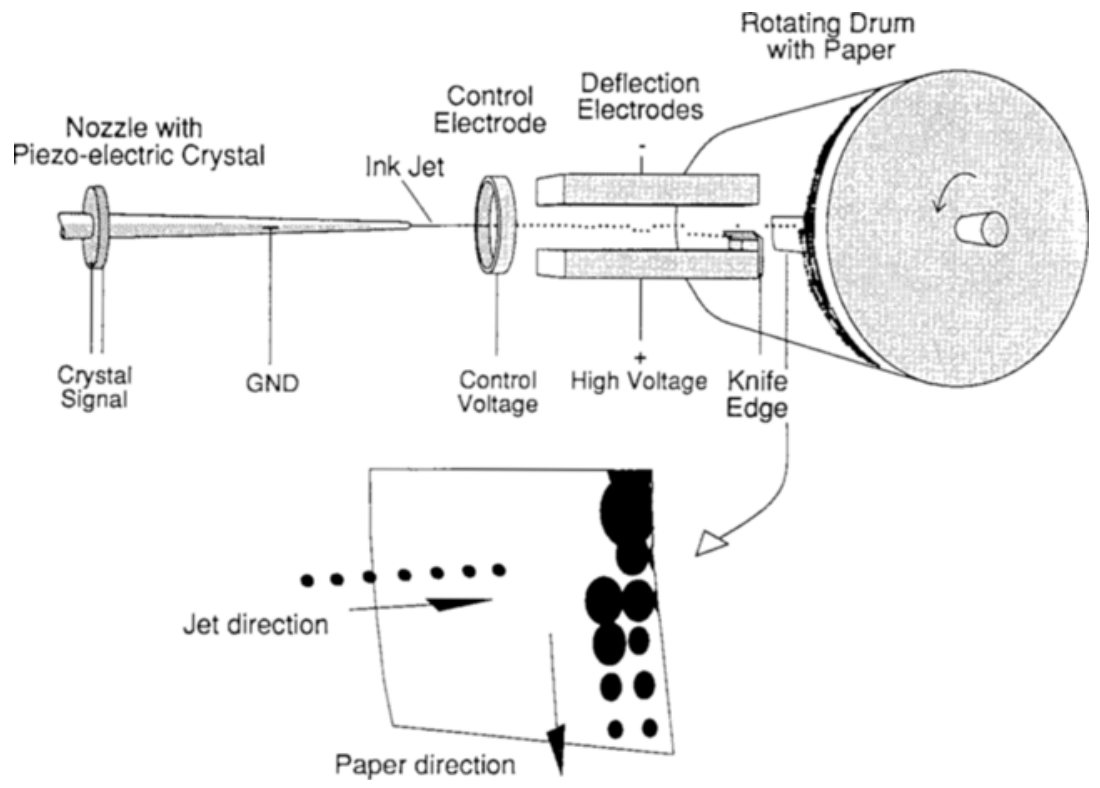

Fig 1. In the ink-jet printer, a continuous jet is ejected from a $10-\mu \mathrm{m}$ nozzle. When the nozzle is stimulated by a piezo-electric crystal, the jet disintegrates into 1 million equally sized drops per second. Each formed drop can be charged by a control electrode located at the point of drop formation. As the drop train enters a static high voltage field generated between the two deflection electrodes, the charged drops are deflected and caught below a knife edge, while the uncharged drops travel unaffected through the deflection field and reach the recording paper. Consequently, by switching the control voltage, the ink-jet can be switched on or off at a maximum frequency equal to that of the drop formation. The diagram at the bottom shows how different gray levels are generated: the printed dot size is varied by controlling the exact number of drops deposited in each pixel. 
through the electrode system toward the recording paper. Thus, depending of whether a single drop is left uncharged, or is charged, it will either reach a pixel on the paper or be prevented from reaching it.

The diagram in the lower portion of Fig 1 shows how different gray levels are produced by the printer. By varying the number of separate drops being deposited in a certain pixel, it is possible to vary the resulting dot size, and thereby the printed density. This true halftone technique makes it possible to produce up to 32 different density levels in every pixel. By combining these discrete levels over a small area of adjacent pixels using an error-diffusion algorithm, ${ }^{7}$ density levels are produced that correspond to data values from 0 to 255 .

For the printing of black-and-white radiographs, a printer has been designed with a printhead consisting of one nozzle (supplied by Siemens-Elema, Solna, Sweden) and an electrode system, like the one described above. The nozzle is fed with black ink (Mingograph writing fluid, Siemens-Elema). The printhead is located in front of the paper-bearing drum and is slowly moved sideways along the drum while it is rotating. The spatial print resolution is 10 pixels/ $\mathrm{mm}$, and the drum dimension is matched to a paper with space for two digital radiographs from the CR system $\left(250 \times 360 \mathrm{~mm}^{2}\right)$, corresponding to the film format of the CR system film recorder. The time required to print a full-size image (two radiographs) is approximately 4.5 minutes.

\section{Adaptation and Processing of Data for the Ink-Jet Printer}

It was not possible to extract the optimized image data from the CR system and transfer it to an external device. Instead, we transferred the nonprocessed raw image data that is stored on the optical disc connected to the CR system. This data transfer was made via a host computer (MicroVax II, Digital Equipment Corp, Maynard, MA) to a Siemens Litebox, a viewing station based on a Macintosh computer, located at the radiology department. From the Litebox, the images were stored on a digital audio tape (DAT). From the DAT, the images were finally entered in the Macintosh computer that con- trols the ink-jet printer at the Department of Electrical Measurements.

Because the ink-jet printed images were to be compared with the film prints, the derived raw image data was level adjusted and processed to give images with comparative information. One part of the image processing was performed by using the commercial software Adobe Photoshop (Adobe Systems Inc, Mountain View, CA) which is capable of handling gray scale images with only 8 bits per pixel. Therefore, the original 10-bit image data was initially converted to 8 bits per pixel. With Adobe Photoshop the image was displayed on the Macintosh monitor, and the window setting was interactively adjusted by an experienced chest radiologist with the goal of obtaining a window setting for evaluation of both the mediastinum and the lungs in the same image.

In the next step, the selected window width was applied to the original 10-bit data, which thereafter was reconverted to 8 bits, ie, the entire usable width was finally represented by values from 0 to 255 .

To further match the image data to that recorded on film, a slight edge enhancement was performed with an unsharp-mask algorithm in Adobe Photoshop. The algorithm was selected to yield a general enhancement of the chest image without obtaining a disturbing noice level.

The above window setting and processing procedures were determined once and for all and then equally applied to all the images.

A gamma curve, corresponding to that of the Macintosh monitor that was used for the window adjustments, was applied to the image data to obtain the printed image as it appeared on the monitor.

Finally, because the ink-jet printer works with discrete drop levels, the 8-bit data was converted to drop numbers for every pixel in a specially developed conversion software.

\section{Ink-Jet Print Substrate}

In the earlier pilot study, ${ }^{1}$ we found a matt coated paper, IJ Mitsubishi NM (Mitsubishi Papermills Ltd, Tokyo, Japan), specially dedicated to ink-jet printing, to be the most suitable print substrate for these type of images. It showed an evenly dense print result with a high 
maximum density. Therefore, this matt coated paper was chosen for the ink-jet prints in this study. These paper prints should be viewed with reflected light.

As stated above, the paper images were printed with a spatial resolution equal to that of the film hard copies, ie, 10 pixels $/ \mathrm{mm}$, giving an image size of $176 \times 214 \mathrm{~mm}^{2}$. The maximum number of drops deposited in a pixel was 25 . With the present ink/paper combination, this gave a maximum reflection density of 1.65 , excluding the density of the white paper.

\section{Evaluation}

Six radiologists (two experienced chest radiologists, three staff members and one resident) reviewed the images. In an earlier study ${ }^{8}$ we noticed that in the mediastinum, only a $12-\mathrm{mm}$ tumor was useful in the evaluation. However, the participants were informed about the size $(6,6,12$, and $18 \mathrm{~mm}$ in the image, due to magnification) and appearance of all the four randomly placed test objects. They were told only to evaluate the presence of the $12-\mathrm{mm}$ nodule in the mediastinum and that of $6 \mathrm{~mm}$ in the right lung. They were also made aware of the fixed objects in the phantom, present in every image (Fig 2).

The observers were instructed to evaluate the ink-jet prints under good light conditions but not at a definitely specified location. To avoid a disturbing background, a special viewing board was prepared: a black light but stiff board of approximately $50 \times 50 \mathrm{~cm}^{2}$ with a white rectangular field in the center, of a size corresponding to the dimension of a single image print (approximately $18 \times 22 \mathrm{~cm}^{2}$ ).

\section{Statistics-ROC Study}

A standard protocol was used with five possible answers: (1) Tumor is certainly present, (2) Tumor is probably present, (3) Tumor is possibly present, (4) Tumor is probably absent, and (5) Tumor is certainly absent.

ROC curve fitting was performed for each region using a computer program (ROCFIT) developed by Metz. ${ }^{2,9}$

The computer program also provides the area $\left(A_{Z}\right)$ under the respective curves. The area is a measure of the general performance of a system. To reduce intraobserver variations we used a method recommended by Metz ${ }^{10}$ whereby the difference of the curve-areas of the different observers was submitted to a paired $t$-test (MS Excel 4.0) to avoid having to repeat the study. In the above tests, a value of $P<.05$ was considered significant.

\section{RESULTS}

The ROC curves for the $12-\mathrm{mm}$ tumor in the mediastinum and the $6-\mathrm{mm}$ nodule in the lung in the different sessions show some divergence for the different observers (numbered 1 through 6; Figs 3 and 4). This is also reflected in the areas $\left(\mathrm{A}_{z}\right)$ under the curves (Table 1 ). As can be seen from Table 1, both film and paper prints resulted in some divergence between the observers. For observers nos. 1 through 5 , the areas under the ROC curves indicate that film performed better than ink-jet printed images. However, for the nodule in the mediastinum, observer 4 was as good on paper as on film. Finally, observer 6 differed from all the others in showing a better performance on ink-jet printed images than on film regarding both the $12-\mathrm{mm}$ nodule in the mediastinum and the lung nodule. On paper, observer 6 had the best results of the six observers; on film the results lie close to the others for the mediastinal nodule whereas the ROC curve area for the pulmonary nodule is relatively small compared with the other observers' curves.

For each of the two objects, the areas under the ROC curves for the six observers were subjected to a two-tailed paired $t$-test. There was no significant difference between the performance of film and ink-jet printed images for the $12-\mathrm{mm}$ tumor in the mediastinum $(P=0.21)$, nor for the small nodule in the right lung $(P=0.31)$.

After averaging the individual observers' responses, "average performance" ROC curves for the ink-jet printed images and the film images were constructed (Fig 5). The curve for film lies above the curve for the ink-jet printed images for both the nodule in the mediastinum and that in the lung. Similarly, the means of the areas under the ROC curves are larger for film than for paper (Table 1). The difference in average performance between film and paper widens if only observers 1 through 5 are included. Judged by the means, the difference in observer performance between film and paper 

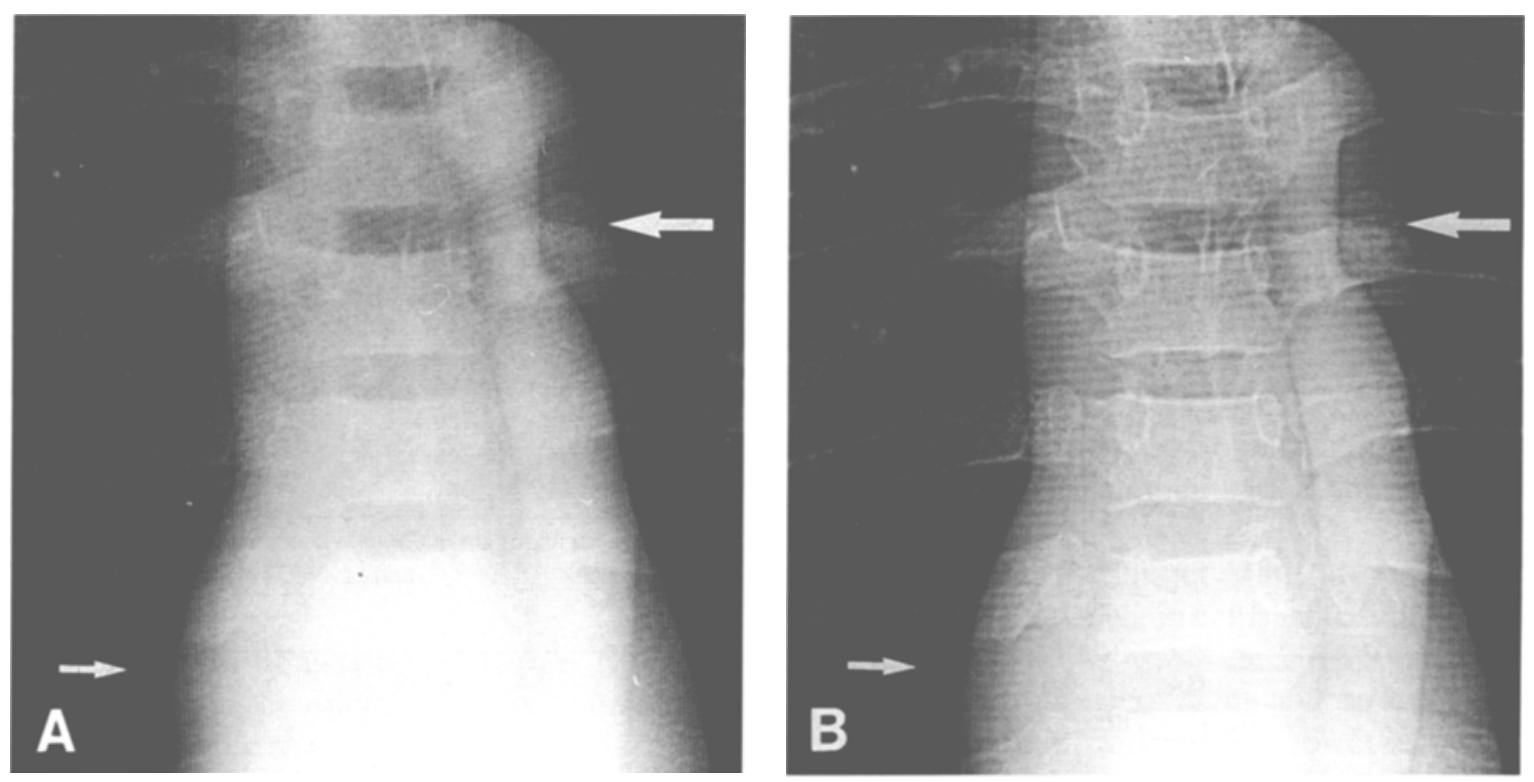

Fig 2. Detail of one of the digital chest phantom radiographs included in the study showing a 6-mm nodule in the right lung (sma/l arrow), and a 12-mm nodule in the mediastinum (largo arrow). (A) film hard copy optimized for lung, (B) film hard copy optimized for the mediastinum, and $\{C\}$ ink-jet printed paper copy

is of the same magnitude for the nodule in the mediastinum and the pulmonary nodule, respectively. Several of the curves had a more or less pronounced "hook" in the upper right corner (Figs 3, 4, and 5).

\section{DISCUSSION}

We found no significant difference in performance between film and ink-jet printed images in this study. This is especially noteworthy

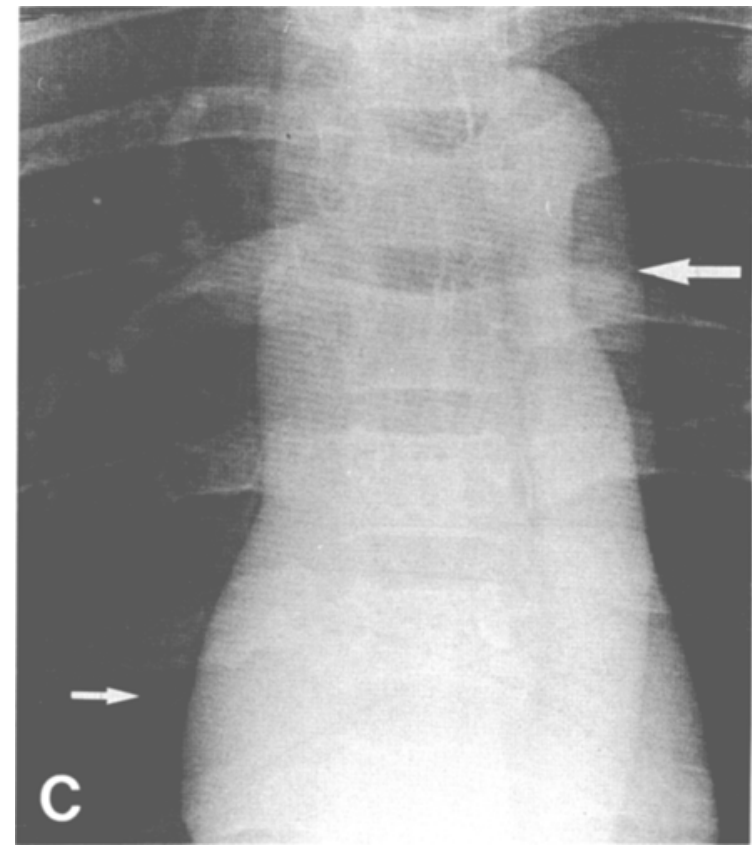

because the observers were given two images on film for each case, optimized for the mediastinum and the lungs, respectively, whereas only one ink-jet printed image was available. The paper image was made to compromise for evaluating both the mediastinum and the lungs. It is our routine at the radiology department to always have two differently processed images on one film. It would, of course, be possible to obtain two differently processed ink-jet printed 

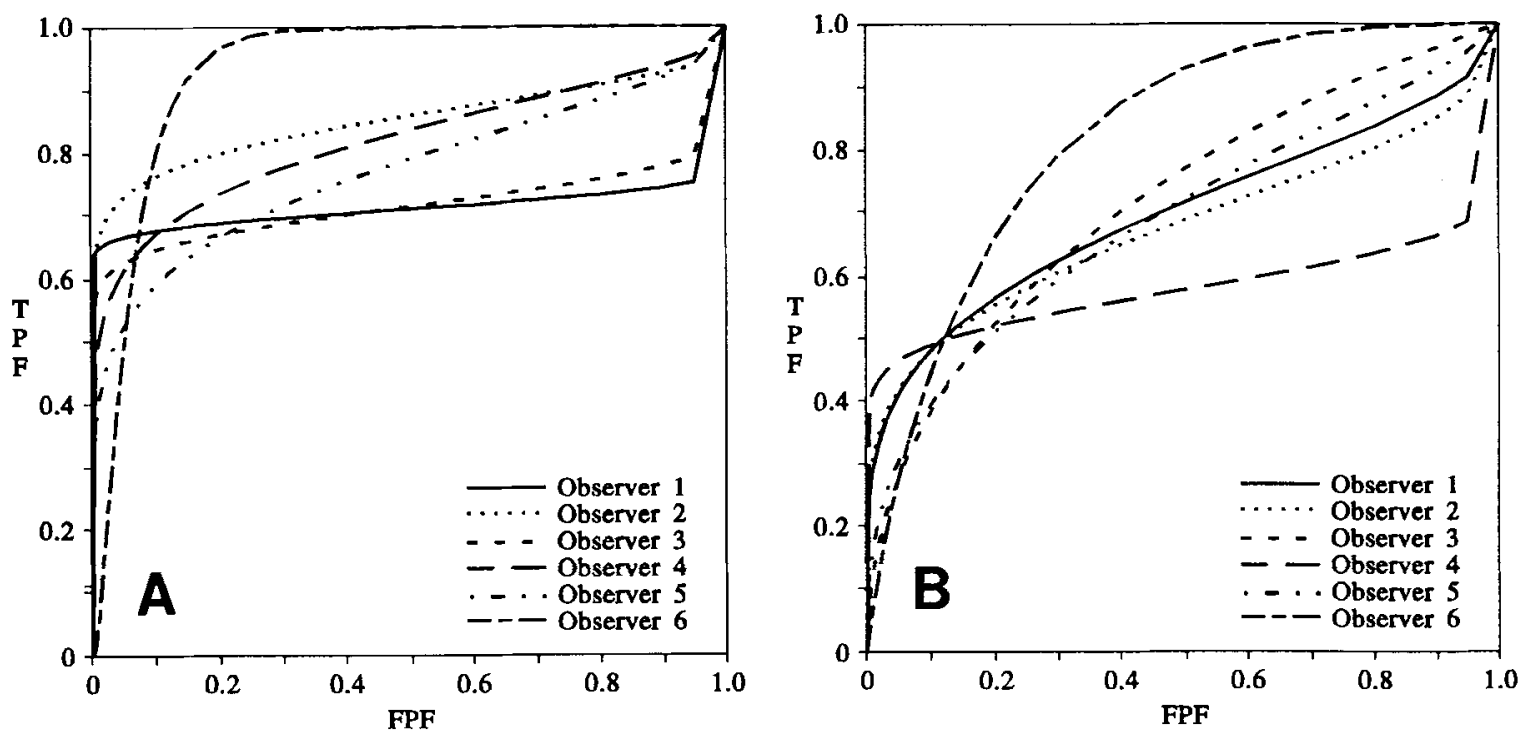

Fig 3. ROC curves for ink-jet printed images showing the difference between the observers, $(A)$ mediastinal tumor and $(B)$ pulmonary tumor. TPF, true-positive fraction; FPF, false-positive fraction.

images as well, but we settled for this compromise in our study, thus further testing the ink-jet printed images. Consequently, this study did not exclusively show the differences between the performances of film and ink-jet printed paper copies as such because differences in image processing were also included. However, from what is described above, it is apparent that this circumstance should be disadvantageous only for the ink-jet printed paper copies. As men-

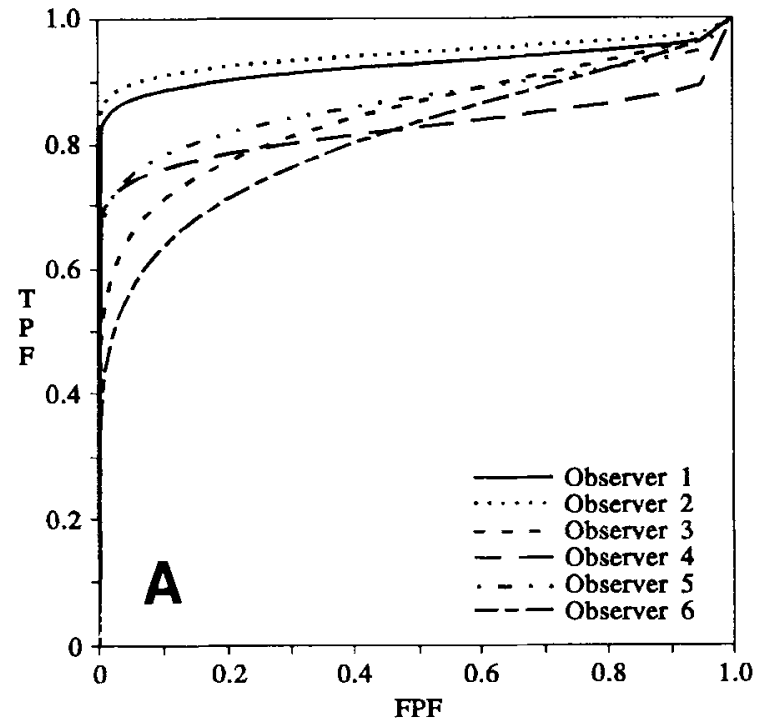

tioned above, observer 6 had better results than the others with paper prints; and the results were also better on paper than on film. In addition, his result with film on the pulmonary nodule seems surprisingly weak compared with the others. (Since he is an experienced staff member this is difficult to explain). Hence, it should be noted that the results would have been less favorable for paper prints if observer 6 had not performed so well on these images

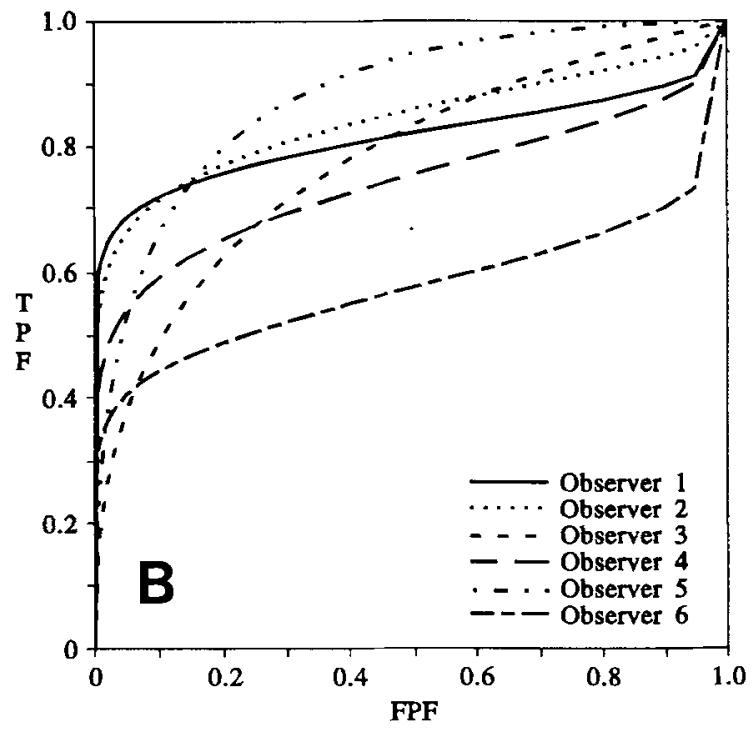

Fig 4. ROC curves for film showing the difference between the observers, $(A)$ mediastinal tumor and $(B)$ pulmonary tumor. TPF, true-positive fraction; FPF, false-positive fraction. 
Table 1. The Areas $\left(A_{z}\right)$ Under the Different Curves From ROCFIT

\begin{tabular}{cccccc}
\hline & \multicolumn{2}{c}{$\begin{array}{c}\text { Mediastinal } \\
\text { "Tumor" }\end{array}$} & & \multicolumn{2}{c}{$\begin{array}{c}\text { Pulmonary } \\
\text { "Tumor" }\end{array}$} \\
\cline { 2 - 3 } \cline { 5 - 6 } Observer & Film & $\begin{array}{c}\text { Paper } \\
\text { Prints }\end{array}$ & Film & $\begin{array}{c}\text { Paper } \\
\text { Prints }\end{array}$ \\
\hline 1 & .92 & .71 & .81 & .70 \\
2 & .94 & .85 & .84 & .68 \\
3 & .85 & .71 & .78 & .72 \\
4 & .82 & .82 & .74 & .58 \\
5 & .87 & .77 & .88 & .69 \\
6 & .81 & .93 & .57 & .82 \\
Mean, 1 through 6 & .87 & .80 & .77 & .70 \\
Mean, 1 through 5 & .88 & .77 & .81 & .67 \\
\hline
\end{tabular}

NOTE: The difference between the areas for all six observers was not significant on a paired $t$-test $(P>.05)$.

compared with film. If the statistical test had included only the other five observers, film would have been shown to perform significantly better than ink-jet printed images.

The hook of some of the curves in the upper right corner has been noted in other studies as well, ${ }^{11}$ and is usually treated as an extrapolation artifact.

In the future, the diagnostic work in radiology departments will most probably be performed on monitors at workstations. Hard copies will only be necessary for demonstration purposes. In that situation, it will be possible to manipulate the monitor image for optimal demonstration of pathology in the individual case and then

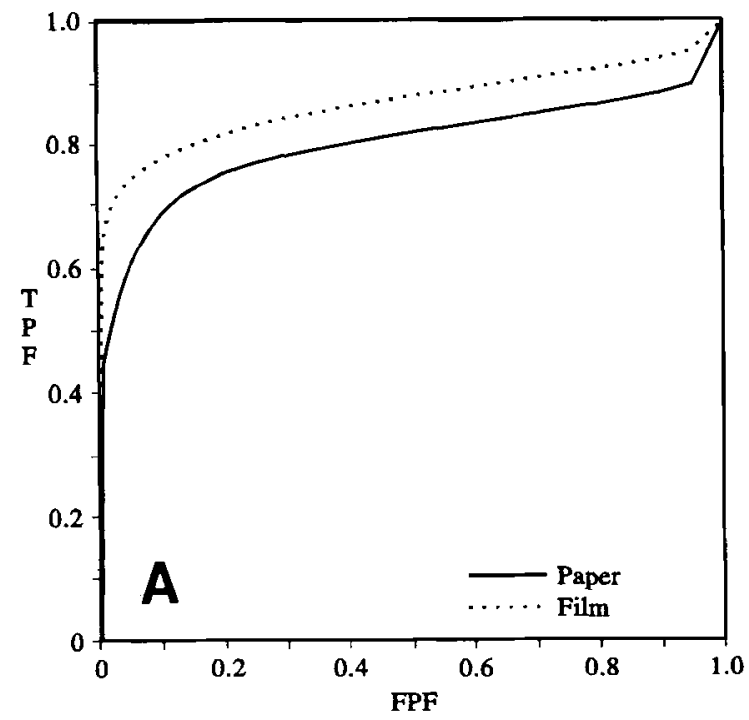

print a copy. Ink-jet printed paper images would be a low cost alternative compared with film, which is expensive because of its high silver content.

Considering that the cost of an ink-jet print on paper is approximately one tenth that of a film print, the economic gain from using ink-jet paper prints will be considerable. The paper copies are so inexpensive that they can be discarded after use. With digital storage of the radiographic images, this will eliminate the present space-consuming and labor-intensive film archives.

The fact that the ink-jet prints are produced as paper images which, in contrast to film prints, are to be viewed with reflected light, gives the viewer the advantage of being able to study the images anywhere, independent of access to a lightbox.

The light fastness of the present ink-jet prints is dependent on the storage situation. Images that are constantly exposed to light will fade in density after a few weeks. On the other hand, images that have been stored in a closed drawer have not shown any changes after a period of 5 years. However, the limited light fastness may not be a major problem; digital storage of the image data and the low cost of each paper print make it possible to produce a new copy when needed.

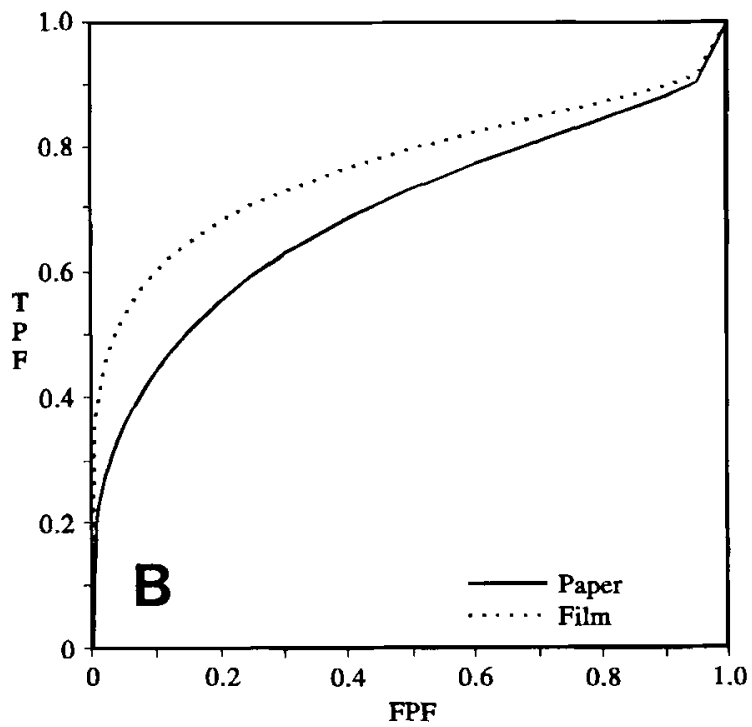

Fig 5. Averaged ROC curves for (A) mediastinum and (B) right lung showing the difference between ink-jet printed images ( - ) and film (‥). TPF, true-positive fraction; FPF, false-positive fraction. 
The printing time for one image is too long compared with that of modern laser printers. However, by using a multinozzle printhead, the time can be reduced by a factor equal to the number of nozzles in use. Nilsson et $\mathrm{al}^{12}$ and Johansson et al $^{13}$ have already described a four-color ink-jet printer with a four-nozzle printhead for the different primary colors cyan, magenta, yellow, and black. With a similar printhead, but with all four nozzles ejecting black ink, the time for printing the full-size image can be reduced to approximately 1 minute, which is less than the time needed to record and develop a film hard copy in the CR system. ${ }^{3}$

The standard chest radiograph is the most commonly performed radiology study, presenting a considerable technical challenge with its wide range of contrast and spatial resolution. The demands on ink-jet printed images will be those of a demonstration medium and not primarily those of diagnostic use. Hence, the goal does not necessarily have to be to make them equal to film in quality. However, our intention is to make the ink-jet printed images as good as possible. Therefore, our results are most encouraging for this new technique.
Although the ROC analysis did not find any difference between ink-jet printed images and film in our present study, there is obvious scope for improvement and the ROC curves could be even closer to each other. We intend to further improve the image quality of the ink-jet printed images in future studies. One important limitation of today's ink-jet printer is the number of true density levels achievable. The present 32 different levels are not enough to produce a smooth scale of gray levels from white to black without losing spatial resolution. This is especially important in the lower density regions, eg, between one and two drops per pixel, where the density differences of consecutive true levels are relatively large. Creating intermediate levels by combining the two nearest levels results in increased noise and decreased spatial resolution in the image. An important step towards improved density reproduction can be taken by combining inks of different densities. Preliminary results have shown that it is possible to reach more than 100 different true density levels by combining two inks. Further studies will show to what extent this feature will improve the quality of the radiographic paper images.

\section{REFERENCES}

1. Kirkhorn T, Kehler M, Nilsson J, et al: Demonstration of digital radiographs by means of ink jet-printed paper copies: Pilot study. J Digit Imaging 5:246-251, 1992

2. Metz CE: Applications of ROC analysis in diagnostic image evaluation, in Haus AG (ed): The Physics of Medical Imaging: Recording System Measurements and Techniques. New York, NY, American Institute of Physics, 1979, pp 546-572

3. Blume H: PCR Technical Review-Articles from previous issues of Market Vision: 1-16. Shelton, CT, Philips Medical Systems, Inc (articles appeared from December 1986-September 1987)

4. Merrit CRB: Computer radiographic imaging using photostimulable phosphors. Mediacamundi 31/2:45-56, 1986

5. Hertz CH, Samuelsson B: Ink jet printing of high quality color images. J Imaging Technol 15:141-148, 1989

6. Kirkhorn $\mathrm{T}$, Nilsson $\mathrm{J}$ : Two true halftone ink jet printers for gray scale and color printing. Report 4/91 Department of Electrical Measurements, Lund Institute of Technology (LUTDEX/TEEM-1042):1-108, 1991
7. Fawcett GS, Schrack GF: Halftoning techniques using error correction. Proc Soc Inform Display 27:305-308, 1986

8. Lyttkens K, Kehler M, Andersson B, et al: Digital luminescence radiography using a chest phantom. Comparison between radiographs displayed on monitor at a workstation and at a personal computer. Acta Radiol 34:440-444, 1993

9. Metz CE: Current problems in ROC analysis. Proceedings of the chest imaging conference 1987. Madison, WI, Medical Physics Publishing Corp, Univ of Wisconsin, 1988

10. Metz CE: ROC methodology in radiologic imaging. Invest Radiol 21:720-733, 1986

11. Metz CE: Some practical issues of experimental design and data analysis in radiological ROC studies. Invest Radiol 24:234-245, 1989

12. Nilsson J, Johansson T, Holmer N-G, et al: Ink jet color printing of digital medical images. Acta Radiol 31:414416,1990

13. Johansson T, Nilsson J, Almquist L-O, et al: The use of ink jets in ultrasound registrations. Ultrasound Med Biol $17: 529-539,1991$ 